\title{
SOME COEFFICIENT PROPERTIES RELATING TO A CERTAIN CLASS OF STARLIKE FUNCTIONS
}

\author{
RAVINDER KRISHNA RAINA AND JANUSZ SOKÓł
}

Received 18 September, 2015

Abstract. This paper considers the problem of determining coefficients in a class $\Delta^{*}$ of normalized starlike functions $f$ analytic in the open unit disk $|z|<1$ satisfying the inequality that

$$
\left|\left\{\frac{z f^{\prime}(z)}{f(z)}\right\}^{2}-1\right|<2\left|\frac{z f^{\prime}(z)}{f(z)}\right| \text {. }
$$

2010 Mathematics Subject Classification: 30C45

Keywords: analytic functions, convex functions, starlike functions, differential subordination

\section{INTRODUCTION}

Let $\mathscr{H}$ denote the class of analytic functions in the open unit disc $\mathbb{U}=\{z:|z|<$ $1\}$ on the complex plane $\mathbb{C}$. Also, let $\mathcal{A}$ denote the subclass of $\mathscr{H}$ comprising of functions $f$ normalized by $f(0)=0, f^{\prime}(0)=1$, and let $\delta \subset \mathcal{A}$ denote the class of functions which are univalent in $\mathbb{U}$. Let a function $f$ be analytic univalent in the unit disc $\mathbb{U}=\{z:|z|<1\}$ on the complex plane $\mathbb{C}$ with the normalization $f(0)=0$, then $f$ maps $\mathbb{U}$ onto a starlike domain with respect to $w_{0}=0$ if and only if

$$
\mathfrak{R e}\left\{\frac{z f^{\prime}(z)}{f(z)}\right\}>0 \quad(z \in \mathbb{U}) .
$$

It is well known that if an analytic function $f$ satisfies $(1.1)$ and $f(0)=0, f^{\prime}(0) \neq 0$, then $f$ is univalent and starlike in $\mathbb{U}$. The set of all functions $f \in \mathcal{A}$ that are starlike univalent in $\mathbb{U}$ will be denoted by $8^{*}$.

For the purpose of this paper, we represent by $\Delta^{*}$ a class which is defined by

$$
\Delta^{*}=\left\{f \in \mathcal{s}^{*}:\left|\left\{\frac{z f^{\prime}(z)}{f(z)}\right\}^{2}-1\right|<2\left|\frac{z f^{\prime}(z)}{f(z)}\right|, z \in \mathbb{U}\right\}
$$

and a related class studied by Rønning [8] was defined by

$$
\delta_{p}=\left\{f \in \delta:\left|\frac{z f^{\prime}(z)}{f(z)}-1\right|<\mathfrak{R e} \frac{z f^{\prime}(z)}{f(z)}, z \in \mathbb{U}\right\} .
$$


Interpreting geometrically the condition in (1.3), we note that $z f^{\prime}(z) / f(z)$ lies inside the parabola

$$
(\mathfrak{I m} w)^{2}<2 \mathfrak{R e} w-1,
$$

and in this way the class $\delta_{p}$ was observed to be connected with certain conic domains. In recent papers $[1-4,6,10]$, certain function classes were considered and were defined under the condition that $z f^{\prime}(z) / f(z)$ lies in a domain which possesses some geometric properties. If we interpret the condition in (1.2) geometrically, then we observe that the product of the distances of $z f^{\prime}(z) / f(z)$ from the foci -1 and 1 is less than twice the distance of $z f^{\prime}(z) / f(z)$ from the origin. The shape of the domain for $Q(z)=z f^{\prime}(z) / f(z)$ is described in Theorem 1 below and the shape of $Q(\mathbb{U})$ is depicted in Figure 1.

Theorem 1 ([9]). If $f(z) \in \Delta^{*}$, then

$$
\mathfrak{R e}\left\{\frac{z f^{\prime}(z)}{f(z)}\right\}>0, \quad z \in \mathbb{U}
$$

and

$$
\left|\frac{z f^{\prime}(z)}{f(z)}-1\right|<\sqrt{2} \text { and }\left|\frac{z f^{\prime}(z)}{f(z)}+1\right|>\sqrt{2}, \quad z \in \mathbb{U} \text {. }
$$

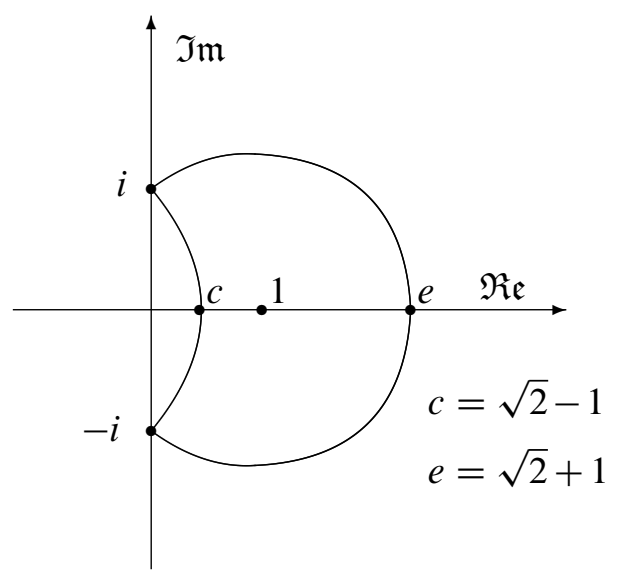

FIGURE 1. The domain for $z f^{\prime}(z) / f(z), f \in \Delta^{*}$. 


\section{COEFFICIENT ESTIMATES}

Theorem 2. If $f(z) \in \Delta^{*}$ and

$$
f(z)=z+\sum_{k=2}^{\infty} a_{k} z^{k}, \quad z \in \mathbb{U}
$$

then

$$
\left|a_{2}\right| \leq 1, \quad\left|a_{3}\right| \leq 3 / 4, \quad\left|a_{4}\right| \leq 1 / 2 .
$$

Proof. In view of (1.2), we have

$$
\left\{\frac{z f^{\prime}(z)}{f(z)}\right\}^{2}-1=2 w(z) \frac{z f^{\prime}(z)}{f(z)}
$$

where

$$
|w(z)|<1 \quad z \in \mathbb{U}, w(z)=\sum_{k=1}^{\infty} c_{k} z^{k} .
$$

Thus, we obtain

$$
\left(z f^{\prime}(z)-f(z)\right)\left(z f^{\prime}(z)+f(z)\right)=2 w(z) z f^{\prime}(z) f(z) .
$$

If we assume that $a_{1}=1$, then from (2.1) and (2.3), we at once have

$$
\begin{aligned}
& \left(\sum_{k=1}^{\infty}(k-1) a_{k} z^{k}\right)\left(\sum_{k=1}^{\infty}(k+1) a_{k} z^{k}\right) \\
= & 2\left(\sum_{k=1}^{\infty} c_{k} z^{k}\right)\left(\sum_{k=1}^{\infty} k a_{k} z^{k}\right)\left(\sum_{k=1}^{\infty} a_{k} z^{k}\right) .
\end{aligned}
$$

Hence, we obtain

$$
\begin{aligned}
& \left(\sum_{k=1}^{\infty}(k+1) a_{k} z^{k}\right)\left(\sum_{k=1}^{\infty}(k-1) a_{k} z^{k}\right) \\
= & \left(2 z+3 a_{2} z^{2}+4 a_{3} z^{3}+\cdots\right)\left(a_{2} z^{2}+2 a_{3} z^{3}+3 a_{4} z^{4}+\cdots\right) \\
= & 2 a_{2} z^{3}+\left(4 a_{3}+3 a_{2}^{2}\right) z^{4}+\left(6 a_{4}+10 a_{2} a_{3}\right) z^{5}+\cdots
\end{aligned}
$$

and

$$
\begin{aligned}
& 2\left(\sum_{k=1}^{\infty} c_{k} z^{k}\right)\left(\sum_{k=1}^{\infty} k a_{k} z^{k}\right)\left(\sum_{k=1}^{\infty} a_{k} z^{k}\right) \\
= & 2\left(c_{1} z+c_{2} z^{2}+c_{3} z^{3}+\cdots\right)\left(z+2 a_{2} z^{2}+3 a_{3} z^{3}+\cdots\right)\left(z+a_{2} z^{2}+a_{3} z^{3}+\cdots\right) \\
= & 2 c_{1} z^{3}+2\left(3 a_{2} c_{1}+c_{2}\right) z^{4}+2\left(4 a_{3} c_{1}+2 a_{2}^{2} c_{1}+3 a_{2} c_{2}+c_{3}\right) z^{5}+\cdots .
\end{aligned}
$$

Equating now the coefficients of like powers of $z$ in (2.6) and (2.7), we have

(i) $a_{2}=c_{1}$, 
(ii) $4 a_{3}+3 a_{2}^{2}=6 a_{2} c_{1}+2 c_{2}$,

(iii) $6 a_{4}+10 a_{2} a_{3}=8 a_{3} c_{1}+4 a_{2}^{2} c_{1}+6 a_{2} c_{2}+2 c_{3}$.

It is well known that the coefficients of the bounded function $w(z)$ satisfies the inequality that $\left|c_{k}\right| \leq 1,(k=1,2,3, \ldots)$, and hence from $(i)$, we have the first inequality of (2.2) that $\left|a_{2}\right| \leq 1$. Now, from ( $i$ ) and ( $\left.i i\right)$, we have

$$
\left|4 a_{3}\right|=2\left|c_{2}+\frac{3}{2} c_{1}^{2}\right| .
$$

Using the estimate (see [7]) that if $w(z)$ has the form (2.3), then

$$
\left|c_{2}-\mu c_{1}^{2}\right| \leq \max \{1,|\mu|\}, \text { for all } \mu \in \mathbb{C},
$$

and we obtain from (2.8) and (2.9) that

$$
\left|a_{3}\right| \leq \frac{3}{4}
$$

which gives the second inequality of of (2.2). From (iii), we find that

$$
\left|6 a_{4}\right|=\left|-10 a_{2} a_{3}+8 a_{3} c_{1}+4 a_{2}^{2} c_{1}+6 a_{2} c_{2}+2 c_{3}\right| .
$$

Because $a_{2}=c_{1}$, (2.10) becomes

$$
\left|6 a_{4}\right|=\left|-2 a_{3} c_{1}+4 c_{1}^{3}+6 c_{1} c_{2}+2 c_{3}\right| .
$$

Moreover, from $(i)-(i i)$, we have

$$
a_{3}=\frac{1}{2} c_{2}+\frac{3}{4} c_{1}^{2},
$$

and from (2.11) and (2.12), we obtain that

$$
\begin{aligned}
\left|6 a_{4}\right| & =\left|-2\left(\frac{1}{2} c_{2}+\frac{3}{4} c_{1}^{2}\right) c_{1}+4 c_{1}^{3}+6 c_{1} c_{2}+2 c_{3}\right| \\
& =\left|\frac{5}{2} c_{1}^{3}+5 c_{1} c_{2}+2 c_{3}\right| \\
& =\left|\frac{5}{2}\left(c_{1}^{3}+2 c_{1} c_{2}+c_{3}\right)-\frac{1}{2} c_{3}\right| .
\end{aligned}
$$

To find the bound for the coefficient $a_{4}$, we next derive some properties of the coefficients $c_{k}$ involved in (2.13). It is known that the function $p(z)$ given by

$$
\frac{1+w(z)}{1-w(z)}=1+p_{1} z+p_{2} z^{2}+\cdots=: p(z)
$$

defines a Caratheodory function with the property that $\mathfrak{R e}\{p(z)\}>0$ in $\mathbb{U}$ and that $\left|p_{k}\right| \leq 2(k=1,2,3, \ldots)$.

Using (2.3) and equating the coefficients of like powers of $z$ in (2.14), we get

$$
p_{2}=2\left(c_{1}^{2}+c_{2}\right) \text { and } p_{3}=2\left(c_{1}^{3}+2 c_{1} c_{2}+c_{3}\right) .
$$


Hence $\left|c_{1}^{2}+c_{2}\right| \leq 1$ and

$$
\left|c_{1}^{3}+2 c_{1} c_{2}+c_{3}\right| \leq 1,
$$

and upon using (2.13) and (2.15), we finally find that

$$
\begin{aligned}
\left|6 a_{4}\right| & \leq\left|\frac{5}{2}\left(c_{1}^{3}+2 c_{1} c_{2}+c_{3}\right)\right|+\left|\frac{1}{2} c_{3}\right| \\
& \leq \frac{5}{2}+\frac{1}{2}=3,
\end{aligned}
$$

which gives the third inequality of (2.2) that $\left|a_{4}\right| \leq 1 / 2$.

Remark. We deem it worthwhile to point out here the sharpness of the estimates of the coefficients given by (2.2) of Theorem 2. Therefore, let us consider the function $f_{1}(z)$ by

$$
\begin{aligned}
f_{1}(z) & =z \exp \int_{0}^{z} \frac{\sqrt{1+t^{2}}+t-1}{t} \mathrm{~d} t \\
& =\frac{2 \sqrt{1+z^{2}}-2}{z} \exp \left\{z-1+\sqrt{1+z^{2}}\right\} \\
& =z+z^{2}+\frac{3}{4} z^{3}+\frac{5}{12} z^{4}+\frac{1}{6} z^{5}+\frac{1}{20} z^{6}+\frac{49}{2880} z^{7} \cdots . \quad(z \in \mathbb{U}) .
\end{aligned}
$$

To show that $f_{1}(z) \in \Delta^{*}$, we need to show that

$$
f_{1}(z) \in 8^{*} \text { and }\left|\left\{\frac{z f_{1}^{\prime}(z)}{f_{1}(z)}\right\}^{2}-1\right|<2\left|\frac{z f_{1}^{\prime}(z)}{f_{1}(z)}\right| .
$$

We note that

$$
0 \leq \mathfrak{R e}\left\{e^{i t}+\sqrt{e^{2 i t}+1}\right\}= \begin{cases}\cos t+\sqrt{|2 \cos t|} \cos t / 2 & \text { for } t \in[0, \pi / 2], \\ \cos t+\sqrt{|2 \cos t|} \sin t / 2 & \text { for } t \in(\pi / 2,3 \pi / 2], \\ \cos t-\sqrt{|2 \cos t|} \cos t / 2 & \text { for } t \in(3 \pi / 2,2 \pi),\end{cases}
$$

and therefore, for the above function $f_{1}$, we have

$$
\mathfrak{R e}\left\{\frac{z f_{1}^{\prime}(z)}{f_{1}(z)}\right\}=\mathfrak{R e}\left\{z+\sqrt{1+z^{2}}\right\}>0 \quad(z \in \mathbb{U}) .
$$

Hence, by (1.1), $f_{1}(z) \in \mathcal{S}^{*}$ and the left-hand side of the second condition in (2.17) becomes

$$
\begin{aligned}
\left|\left\{\frac{z f_{1}^{\prime}(z)}{f_{1}(z)}\right\}^{2}-1\right| & =\left|\left\{z+\sqrt{1+z^{2}}\right\}^{2}-1\right| \\
& =2|z|\left|z+\sqrt{1+z^{2}}\right| \\
& <2\left|z+\sqrt{1+z^{2}}\right|
\end{aligned}
$$




$$
=2\left|\frac{z f_{1}^{\prime}(z)}{f_{1}(z)}\right|,
$$

which implies that $f_{1}(z) \in \Delta^{*}$. Thus, from (2.16), we see that the first and the second estimations in (2.2) are sharp. The question is whether the third estimation $\left|a_{4}\right| \leq 1 / 2$ is sharp in the class. The function (2.16) suggest the following conjecture.

Conjecture 1. If $f(z)$ defined by (2.18) belongs to $\Delta^{*}$, then

$$
\left|a_{4}\right| \leq \frac{5}{12}=0.416 \ldots
$$

In the sequel, we find somewhat weaker estimation for $\left|a_{n}\right|$, for the next coefficients too by applying another method.

Theorem 3. If $f(z) \in \Delta^{*}$ and

$$
f(z)=z+\sum_{k=2}^{\infty} a_{k} z^{k}, \quad z \in \mathbb{U},
$$

then for $n=2,3,4, \ldots$, we have

$$
(n-1)^{2}\left|a_{n}\right|^{2} \leq \sum_{k=1}^{n-1}\left|a_{k}\right|^{2}\left(1+2 k-k^{2}\right) .
$$

Proof. In view of (1.5), we have

$$
\frac{z f^{\prime}(z)}{f(z)}-1=\sqrt{2} w(z)
$$

where

$$
|w(z)|<1 \quad z \in \mathbb{U}, \quad w(z)=\sum_{k=1}^{\infty} c_{k} z^{k}
$$

Thus, we obtain

$$
\frac{1}{\sqrt{2}}\left(z f^{\prime}(z)-f(z)\right)=w(z) f(z)
$$

and from (2.18) and (2.20), we at once have

$$
\frac{1}{\sqrt{2}} \sum_{k=1}^{\infty}(k-1) a_{k} z^{k}=\sum_{k=1}^{\infty} c_{k} z^{k} \sum_{k=1}^{\infty} a_{k} z^{k}, a_{1}=1 .
$$

Thus, we get

$$
\frac{1}{\sqrt{2}} \sum_{k=1}^{n}(k-1) a_{k} z^{k}+\frac{1}{\sqrt{2}} \sum_{k=n+1}^{\infty}(k-1) a_{k} z^{k}=w(z)\left\{\sum_{k=1}^{n-1} a_{k} z^{k}+\sum_{k=n}^{\infty} a_{k} z^{k}\right\}
$$


which gives

$$
\sum_{k=1}^{n} \frac{k-1}{\sqrt{2}} a_{k} z^{k}+\sum_{k=n+1}^{\infty} \frac{k-1}{\sqrt{2}} a_{k} z^{k}-\sum_{k=1}^{\infty} c_{k} z^{k} \sum_{k=n}^{\infty} a_{k} z^{k}=w(z)\left\{\sum_{k=1}^{n-1} a_{k} z^{k}\right\} .
$$

Therefore, we can write

$$
\sum_{k=1}^{n} \frac{k-1}{\sqrt{2}} a_{k} z^{k}+\sum_{k=n+1}^{\infty} b_{k} z^{k}=w(z) \sum_{k=1}^{n-1} a_{k} z^{k}
$$

for some $b_{k}, n+1 \leq k<\infty$, where $b_{k}$ can be expressed in terms of the following relation involving the coefficients $a_{k}$ and $c_{k}$ :

$$
b_{k}=\frac{k-1}{\sqrt{2}} a_{k}-\sum_{j=1}^{k-n} c_{j} a_{k-j} .
$$

This gives

$$
\begin{aligned}
\left|\sum_{k=1}^{n} \frac{k-1}{\sqrt{2}} a_{k} z^{k}+\sum_{k=n+1}^{\infty} b_{k} z^{k}\right|^{2} & =\left|w(z) \sum_{k=1}^{n-1} a_{k} z^{k}\right|^{2} \\
& \leq\left|\sum_{k=1}^{n-1} a_{k} z^{k}\right|^{2},
\end{aligned}
$$

where

$$
\sum_{k=1}^{n} \frac{k-1}{\sqrt{2}} a_{k} z^{k}+\sum_{k=n+1}^{\infty} b_{k} z^{k}:=\sum_{k=1}^{\infty} d_{k} z^{k}
$$

is an analytic function in the unit disc. Making use of the known formula (see, for instance [5])

$$
\int_{0}^{2 \pi}\left|\sum_{k=1}^{\infty} d_{k}\left(r e^{i \theta}\right)^{k}\right|^{2} d \theta=2 \pi \sum_{n=1}^{\infty}\left|d_{n}\right|^{2} r^{2 n}
$$

and integrating on $z=r e^{i \theta}, 0<r<1,0 \leq \theta<2 \pi$, both the sides of (2.22), we obtain

$$
\sum_{k=1}^{n} \frac{(k-1)^{2}}{2}\left|a_{k}\right|^{2} r^{2 k}+\sum_{k=n+1}^{\infty}\left|b_{k}\right|^{2} r^{2 k} \leq \sum_{k=1}^{n-1}\left|a_{k}\right|^{2} r^{2 k}
$$

Therefore,

$$
\frac{1}{2} \sum_{k=1}^{n}(k-1)^{2}\left|a_{k}\right|^{2} r^{k} \leq \sum_{k=1}^{n-1}\left|a_{k}\right|^{2} r^{2 k}
$$


which upon letting $r \rightarrow 1$ gives

$$
\frac{1}{2} \sum_{k=1}^{n}(k-1)^{2}\left|a_{k}\right|^{2} \leq \sum_{k=1}^{n-1}\left|a_{k}\right|^{2},
$$

and this leads to the desired result (2.19).

Theorem 4. Let $f(z)$ defined by (2.18) belong to $\Delta^{*}$. Then for $n \geq 3$, we have

$$
\left|a_{n}\right| \leq \frac{\sqrt{3}}{n-1}
$$

Proof. From (2.19), we have (for $n \geq 3$ )

$$
\begin{aligned}
(n-1)^{2}\left|a_{n}\right|^{2} & \leq \sum_{k=1}^{n-1}\left|a_{k}\right|^{2}\left(1+2 k-k^{2}\right) \\
& =2\left|a_{1}\right|^{2}+\left|a_{2}\right|^{2}-2\left|a_{3}\right|^{2}-7\left|a_{4}\right|^{2}-\ldots \\
& \leq 2\left|a_{1}\right|^{2}+\left|a_{2}\right|^{2} \\
& =2+\left|a_{2}\right|^{2} .
\end{aligned}
$$

Furthermore, $\left|a_{2}\right|^{2} \leq 1$ by Theorem 2, and we have then

$$
(n-1)^{2}\left|a_{n}\right|^{2} \leq 3
$$

and finally we obtain (2.23).

\section{ACKNOWLEDGEMENT}

The authors would like to express their sincerest thanks to the referees for a careful reading and various suggestions made for the improvement of the paper.

\section{REFERENCES}

[1] J. Dziok, R. K. Raina, and J. Sokół, "Certain results for a class of convex functions related to a shell-like curve connected with Fibonacci numbers," Comput. Math. Appl., vol. 61, no. 9, pp. 2605-2613, 2011, doi: 10.1016/j.camwa.2011.03.006.

[2] J. Dziok, R. K. Raina, and J. Sokół, "On alpha-convex functions related to shell-like functions connected with Fibonacci numbers," Appl. Math. Comput., vol. 218, pp. 966-1002, 2011, doi: 10.1016/j.amc.2011.01.059.

[3] J. Dziok, R. K. Raina, and J. Sokół, "Differential subordinations and alpha-convex functions," Acta Math. Scientia, vol. 33B, pp. 609-620, 2013, doi: 10.1016/S0252-9602(13)60024-7.

[4] J. Dziok, R. K. Raina, and J. Sokół, "On a class of starlike functions related to a shell-like curve connected with Fibonacci numbers," Math. Comput. Modelling, vol. 57, pp. 1203-1211, 2013.

[5] A. W. Goodman, Univalent Functions, Vol. I. Tampa, Florida: Mariner Publishing Co., 1983.

[6] R. Jurasińnska and J. Sokół, "Some problems for certain family of starlike functions," Math. Comput. Modelling, vol. 55, pp. 2134-2140, 2012, doi: 10.1016/j.mcm.2012.01.009.

[7] F. R. Keogh and E. P. Merkes, "A coefficient inequality for certain classes of analytic functions," Proc. Amer. Math. Soc., vol. 20, pp. 8-12, 1969, doi: 10.2307/2035949. 
[8] F. R. Keogh and E. P. Merkes, "Uniformly convex functions and a corresponding class of starlike functions," Proc. Amer. Math. Soc., vol. 118, pp. 189-196, 1993.

[9] R. K. Raina and J. Sokól, "Some properties relating to a certain class of starlike functions," Comptes Rendus Mathématique, vol. 353, no. 11, pp. 973-978, 2015.

[10] J. Sokół and A. Wiśniowska-Wajnryb, "On certain problem in the class of $k$-starlike functions," Comput. Math. Appl., vol. 62, pp. 4733-4741, 2011, doi: 10.1016/j.camwa.2011.10.064.

\section{Authors' addresses}

\section{Ravinder Krishna Raina}

M.P. University of Agriculture and Technology, Department of Mathematics, 10/11 Ganpati Vihar, Opposite Sector 5, Udaipur 313002, Rajasthan, India

E-mail address: rkraina_7@hotmail.com

\section{Janusz Sokół}

University of Rzeszów, Faculty of Mathematics and Natural Sciences, ul. Prof. Pigonia 1, 35-310 Rzeszów, Poland

E-mail address: jsokol@ur.edu.pl 